\section{Tissue culture efficiency of wheat species with different genomic formulas}

\author{
Olga Alikina ${ }^{1}$, Mariya Chernobrovkina ${ }^{2}$, Sergey Dolgov ${ }^{1,2}$ and \\ Dmitry Miroshnichenko ${ }^{1,2^{*}}$
}

\begin{abstract}
Ancient wheats are increasingly considered as valuable resources for genes of interest which could be analyzed and introduced into cultivated varieties by genetic engineering technologies. The first stage of biotechnological crop improvement consists of successful in vitro plant regeneration. Twelve wheat germplasms with different genomic formulas ( $A A, A A B B, A A G G, A A B B D D, A A D$ $D G G$ genomes) were examined with the use of two explant types (immature vs. mature embryos). All of the tested germplasms were able to regenerate plants, although the morphogenic ability of immature embryos was higher. The highest rate of embryogenic/regenerable structure formation was found in immature embryo cultures of tetraploid species (T. polonicum, T. turgidum, T. carthlicum, and T. dicoccum) as well as of hexaploid T. spelta. At the same time, diploid einkorn wheat (T. monococcum) and polyploid species with $G$ chromosomes (T. timopheevii and T. kiharae) were characterized by low embryogenesis and by the presence of albino plantlets among shoots.
\end{abstract}

Key words: Callus induction, somatic embryogenesis, plant regeneration, albino plants.

\section{INTRODUCTION}

Wheat (Triticum L.) is one of the most important food grains used around the globe for human food and livestock feed. Over the last decade, wild and cultured ancient wheats were increasingly involved in modern wheat breeding programs, as donors of genes conferring resistance to both biotic and abiotic stresses (Nevo 2011, Longin and Reif 2014). However, the exploitation of these resources is time-consuming and limited by cross-incompatibility barriers and linkage drags. In addition, the problem of determining the function of genes responsible for enhanced levels of resistance/tolerance is an obstacle. The transgenic approach, along with the exploration of natural or artificial mutant phenotypes, may be used as a powerful tool to detect, reduce or knock out the expression of candidate genes to clarify their functions (Repellin et al. 2001). Thereafter, this biotechnological tool can accelerate wheat breeding by the creation of artificially improved cis-genic lines, by introducing the discovered genes of interest into the existing cultivar, avoiding interspecific crossing barriers. Unfortunately, transgenic studies are basically focused on the two most common species: bread wheat (T. aestivum L., AABBDD genome) and pasta (durum) wheat (T. turgidum L., AABB genome), which is the reason for the current lack of genetic transformation protocols for ancient cultivated wheats (Mamrutha et al. 2014, Jones 2015).
Crop Breeding and Applied Biotechnology 16: 307-314, 2016 Brazilian Society of Plant Breeding. Printed in Brazil http://dx.doi.org/10.1590/1984$70332016 v 16 n 4 a 46$ (n)

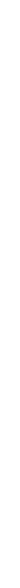


In the development of wheat species transformation techniques, the critical factor is the capacity of routine regeneration of whole plants from in vitro tissue cultures. Wheat includes more than 20 cultivated species (Goncharov 2011), however substantial research effort has been dedicated mainly to durum and bread wheat, owing to their prevalence in the world production (Fennel et al. 1996, Machii et al. 1998, Barro et al. 1999, Bohorova et al. 2001, He and Lazzeri 2001, Zale et al. 2004, Vendruscolo et al. 2008, Yin et al. 2011). Until recently, many wheat species were not analyzed for plant regeneration ability in vitro; but nowadays, the interest in such studies is on the rise (Chang et al. 2012, Yang et al. 2015, Özgen et al. 2015). Over the years, several studies have demonstrated the possibility to regenerate plants from different tissues of tetraploid emmer wheat (AABB genome) (Eapen and Rao 1982, Chauhan et al. 2007, Bi et al. 2007, Chang et al. 2012, Yang et al. 2015), diploid T. monococcum and T. urartu (AA genome) (Eudes et al. 2003, Yang et al. 2015). Proliferation was induced in somatic cell cultures of tetraploid timopheevii wheat (AAGG genome) and hexaploid spelt wheat (AABBDD genome), although no shoot differentiation was observed (Lazar et al. 1983) or the number of regenerated plants was low (Yang et al 2015, Özgen et al. 2015). Some attempts to establish reliable plant regeneration protocols using various tissues of diploid wheat species (AA genome) failed to produce positive results (Lazar et al. 1983, Zale et al. 2004, Bi et al. 2007, Yin et al. 2011). According to Yang et al. (2015), plantlet regeneration by means of somatic embryogenesis represents a great obstacle for various germplasms, e.g., T. carthlicum, T. macha and T. polonicum. Thus, even after many years of research, during which various tissues, culture media and environmental conditions were tested, the screening of in vitro response of germplasms/genotypes is still important for biotechnological applications.

Immature embryos are currently the most frequently and successfully used explants for the initiation of regeneration of wheat species (Machii et. al 1998, Barro et al. 1999, Bohorova et al. 2001, He and Lazzeri 2001, Pellegrineschi et al. 2002, Eudes et al. 2003, Vendruscolo et al. 2008, Miroshnichenko et al. 2013) and for reliable and efficient genetic engineering (Mamrutha et al. 2014, Jones 2015). However, the cultivation of donor plants to ensure a regular supply of immature embryos is labor-intensive and requires a lot of time and space. Mature dry seeds can be used as alternative explants, providing low-cost and year-round accessibility. Efficient in vitro culture of mature wheat embryos was established by two main techniques. Embryos should be isolated from mature in the same way as from immature seeds, followed by placing the entire (Chauhan et al. 2007, Özgen et al. 2015), or longitudinally bisected (Zale et al. 2004, Yu et al. 2008) or multiple divided embryos (Delporte et al. 2014) on callus induction medium. Alternatively, in vitro plant regeneration under endosperm-supported culture may be achieved by placing the whole seed with the wounded mature embryo on induction medium (Özgen et al. 1998, Filippov et al. 2006).

The aim of the current investigation was to analyze a set of promising wheat germplasms with different genomic formulas (genomes $A A, A A B B, A A G G, A A B B D D, A A D D G G$ ) in order to clarify which kind of germplasms might have direct application in molecular breeding and genetic transformation programs and which ones require further optimization of the in vitro regeneration technique. To this end, the immature embryo cultures and endosperm-supported mature embryo cultures were compared.

\section{MATERIAL AND METHODS}

Immature and mature embryo tissues of 12 spring wheat germplasms, namely diploid $(2 n=2 x=14)$, tetraploid $(2 n=4 x=28)$ and hexaploid $(2 n=6 x=42)$ were used. Diploid wheat species were T. monococcum L. (einkorn, $A A)$ and T. sinskajae A.Filat. et Kurk (sinskajae wheat, AA). Tetraploid wheat species were T. dicoccum (Schrank) Schubl. (emmer wheat 'Runo', AABB), T. carthlicum Nevski (Persian wheat, AABB), T. turgidum L. (cone wheat, AABB), T. polonicum L. (Polish wheat, AABB) and T. timopheevii Zhuk (timopheevii wheat, AAGG). Hexaploid wheat species were T. spelta L. (spelt wheat, AABBDD), two germplasms of T. compactum Host. (white and red club wheats, AABBDD), T. sphaerococcum Perciv. (shot wheat, AABBDD) and T. kiharae Dorof. et Migusch (kiharae wheat, AADDGG).

To induce plant regeneration from immature embryo culture we used a conventional two-step protocol that included 4-week cultivation of explants in the dark on media containing 2,4-D (2,4-dichlorophenoxyacetic acid) following plant differentiation in the light on media without growth regulators (Fennel et al. 1996, Machii et al. 1998, Pellegrineschi et al. 2002, Chauhan et al. 2007, Miroshnichenko et al. 2013). Immature embryos were isolated from caryopses of greenhousegrown wheat plants 11-15 days after anthesis. The caryopses were placed in $70 \%$ ethanol solution for 3 min, thereafter soaked for $20-25 \mathrm{~min}$ in a $20 \%$ solution of commercial bleach ( $5.25 \%$ sodium hypochlorite) and then rinsed in sterile distilled water. Wheat embryos with a size of 1-2 mm were extracted under a binocular microscope, with subsequent 
placement on callus induction medium, scutellum side up. After 30 days of culturing at $25^{\circ} \mathrm{C}$ in the dark, the number of explants that produced embryogenic/nonembryogenic callus was scored. Endosperm-supported mature embryo culture was performed according to a protocol based on the combined application of 3,6-dichloro-o-anisic acid (Dicamba) and indoleacetic acid (IAA), for embryogenic callus induction (Filippov et al. 2006, Miroshnichenko et al. 2011). Prepared seeds were placed furrow down in Petri dishes and incubated at $25^{\circ} \mathrm{C}$ in the dark for 25 days. After that the number of explants producing embryogenic/nonembryogenic callus was scored. The callus induction medium for immature embryos contained mineral salts and vitamins according to Murashige and Skoog (1962) and was supplemented with $30 \mathrm{~g} \mathrm{~L}^{-1}$ sucrose, $150 \mathrm{mg} \mathrm{L}^{-1}$ asparagine, $7 \mathrm{~g} \mathrm{~L}^{-1}$ agarose, and $2 \mathrm{mg} \mathrm{L}^{-1}$ 2,4-D. The medium for callus induction from mature embryo tissues contained the same mineral salts and vitamins, $20 \mathrm{~g} \mathrm{~L}^{-1}$ sucrose, $7 \mathrm{~g} \mathrm{~L}^{-1}$ agarose, $12 \mathrm{mg} \mathrm{L}^{-1}$ Dicamba, and $0.1 \mathrm{mg} \mathrm{L}^{-1}$ IAA. For plant regeneration, embryogenic calli produced by mature or immature embryos were transferred into culture flasks on hormone-free medium containing MS mineral salts and vitamins, $20 \mathrm{~g} \mathrm{~L}^{-1}$ sucrose, and $7 \mathrm{~g} \mathrm{~L}^{-1}$ agarose. Calli were cultured under a photoperiod of $16 \mathrm{~h} / 8 \mathrm{~h}$ (day/night) for 30 days at $26^{\circ} \mathrm{C}$. At the end of culturing, the number of plantlets regenerated from embryogenic calli was calculated.

Each experiment was considered a completely randomized design. The treatments were repeated at least three times. Each Petri dish was considered one replication. Every dish contained 10-12 mature seeds or 20-23 immature embryos. Statistical analysis was performed with the use of ANOVA and Duncan's Multiple Range Test (determination of the significance of results with the use of $\mathrm{LSD}_{05}$ ). We analyzed the frequency of callus induction per 100 explants, the frequency of embryogenic callus induction per 100 explants, the frequency of callus regeneration per 100 explants, the number of regenerated shoots per one embryogenic callus and the number of albino shoots per one embryogenic callus. On the basis of these indices, we calculated in vitro culture efficiency as the number of produced green shoots per one initial explant. Software Statistica 10.0 was used for statistical calculations.

\section{RESULTS AND DISCUSSION}

Callus growth and embryogenesis were observed in the cultured tissues of all tested wheat species (Tables 1 and 2). The appearance of primary calli on the explant surface was observed after two to four days of culturing. Mixtures of the embryogenic callus surrounded by amorphous callus were observed in most of the wheat species after 10-15 days of cultivation (Figure 1). Non-regenerating amorphous callus was friable, soft and translucent. Immature embryo tissues produced yellowish to white, nodular embryogenic callus, characterized by the formation of leaf-like structures, green shoots and rooted shoots after transferring on the regeneration medium. Unlike the immature embryos, in which the scutellar tissue was clearly identified as a source for embryogenesis, the embryogenic callus of mature embryos was originated from divided cells of the coleoptile base, first node and epiblast. Therefore the embryogenic callus of

Table 1. In vitro culture response of immature embryo tissue of different wheat species ${ }^{1}$

\begin{tabular}{|c|c|c|c|c|c|c|c|c|}
\hline \multirow{2}{*}{ Species } & \multirow{2}{*}{ Genome } & \multirow{2}{*}{$\begin{array}{l}\text { No. of } \\
\text { cultured } \\
\text { explants }\end{array}$} & \multirow{2}{*}{$\begin{array}{c}\text { Callus } \\
\text { induction } \\
(\%)^{2}\end{array}$} & \multirow{2}{*}{$\begin{array}{c}\text { Embryogenic } \\
\text { callus } \\
\text { induction } \\
(\%)^{3}\end{array}$} & \multirow{2}{*}{$\begin{array}{l}\text { Regeneration } \\
\text { efficiency } \\
(\%)^{4}\end{array}$} & \multicolumn{2}{|c|}{$\begin{array}{c}\text { No. of regenerated } \\
\text { shoots }^{5}\end{array}$} & \multirow{2}{*}{$\begin{array}{l}\text { Culture } \\
\text { efficiency }\end{array}$} \\
\hline & & & & & & Total & $\begin{array}{l}\text { Albino } \\
\text { plants }\end{array}$ & \\
\hline T. sinskajae (sinskajae wheat) & A & 115 & $93.9 \mathrm{ab}$ & $83.5 \mathrm{c}$ & $82.6 \mathrm{c}$ & 6.9 e & $0.22 \mathrm{~b}$ & $5.5 f$ \\
\hline T. dicoccum (emmer wheat 'Runo') & $A B$ & 150 & $97.3 \mathrm{ab}$ & $94.0 \mathrm{ab}$ & $94.0 \mathrm{ab}$ & $12.8 \mathrm{c}$ & $0.00 \mathrm{~b}$ & $12.0 \mathrm{bc}$ \\
\hline T. carthlicum (persian wheat) & $A B$ & 183 & $97.8 \mathrm{ab}$ & $86.3 \mathrm{bc}$ & $86.3 \mathrm{bc}$ & $16.4 \mathrm{ab}$ & $0.01 \mathrm{~b}$ & $14.1 \mathrm{a}$ \\
\hline T. turgidum (cone wheat) & $A B$ & 192 & 85.4 bc & $82.8 \mathrm{c}$ & $81.8 \mathrm{c}$ & $15.2 \mathrm{~b}$ & $0.00 \mathrm{~b}$ & $12.3 \mathrm{~b}$ \\
\hline T. timopheevii (timopheevii wheat) & AG & 140 & $77.1 \mathrm{c}$ & $14.3 \mathrm{~h}$ & $12.9 \mathrm{~h}$ & 7.4 e & $0.39 \mathrm{~b}$ & $0.9 \mathrm{~h}$ \\
\hline T. compactum (white club wheat) & $A B D$ & 227 & $86.3 \mathrm{bc}$ & $63.4 \mathrm{e}$ & $63.4 \mathrm{e}$ & $12.4 \mathrm{c}$ & $0.00 \mathrm{~b}$ & 7.9 e \\
\hline T. compactum (red club wheat) & $A B D$ & 158 & $94.3 \mathrm{ab}$ & $54.4 \mathrm{f}$ & $54.4 \mathrm{f}$ & $17.5 \mathrm{a}$ & $0.10 \mathrm{~b}$ & $9.4 \mathrm{~d}$ \\
\hline T. spelta (spelt wheat) & $A B D$ & 179 & $97.2 \mathrm{ab}$ & $73.2 \mathrm{~d}$ & $73.2 \mathrm{~d}$ & $17.2 \mathrm{a}$ & $0.00 \mathrm{~b}$ & $12.6 \mathrm{~b}$ \\
\hline
\end{tabular}

${ }^{1}$ Different letters, in each column, indicate statistical differences by the Duncan's Multiple Range Test $(p<0.05) ;{ }^{2}$ Percentage of initial explants produced callus; ${ }^{3}$ Percentage of initial explants produced embryogenic callus; ${ }^{4}$ Percentage of initial explants produced plantlets after the transferring to regeneration medium; ${ }^{5}$ Number of regenerated plantlets per one embryogenic callus; ${ }^{6}$ Average number of green plants regenerated per one initial explant. 
Table 2. In vitro culture response of mature embryo tissue of different wheat species

\begin{tabular}{|c|c|c|c|c|c|c|c|c|}
\hline \multirow{2}{*}{ Species } & \multirow{2}{*}{ Genome } & \multirow{2}{*}{$\begin{array}{l}\text { No. of } \\
\text { cultured } \\
\text { explants }\end{array}$} & \multirow{2}{*}{$\begin{array}{c}\text { Callus } \\
\text { induction } \\
(\%)^{2}\end{array}$} & \multirow{2}{*}{$\begin{array}{c}\text { Embryogenic } \\
\text { callus } \\
\text { induction } \\
(\%)^{3} \\
\end{array}$} & \multirow{2}{*}{$\begin{array}{c}\text { Regeneration } \\
\text { efficiency } \\
(\%)^{4}\end{array}$} & \multicolumn{2}{|c|}{$\begin{array}{c}\text { No. of regenerated } \\
\text { shoots }^{5}\end{array}$} & \multirow{2}{*}{$\begin{array}{l}\text { Culture } \\
\text { efficiency }^{6}\end{array}$} \\
\hline & & & & & & Total & $\begin{array}{l}\text { Albino } \\
\text { plants }\end{array}$ & \\
\hline T. sinskajae (sinskajae wheat) & A & 82 & 100.0 & $8.5 \mathrm{~cd}$ & $6.1 \mathrm{cdef}$ & $6.2 \mathrm{e}$ & 0.0 & $0.38 \mathrm{e}$ \\
\hline T. dicoccum (emmer wheat 'Runo') & $A B$ & 161 & 100.0 & $28.6 \mathrm{a}$ & $28.6 \mathrm{a}$ & $11.9 \mathrm{~b}$ & 0.0 & $3.41 \mathrm{a}$ \\
\hline T. turgidum (cone wheat) & $A B$ & 186 & 100.0 & $4.3 \mathrm{de}$ & 3.8 def & $8.3 \mathrm{~cd}$ & 0.0 & 0.31 ef \\
\hline T. timopheevii (timopheevii wheat) & AG & 172 & 98.8 & $12.2 \mathrm{bc}$ & $11.0 \mathrm{~cd}$ & 6.5 de & 0.0 & $0.72 \mathrm{~d}$ \\
\hline T. compactum (white club wheat) & $A B D$ & 181 & 100.0 & $6.1 \mathrm{cde}$ & 4.4 def & $8.5 \mathrm{c}$ & 0.0 & $0.38 \mathrm{e}$ \\
\hline T. compactum (red club wheat) & $A B D$ & 158 & 100.0 & $5.1 \mathrm{de}$ & 4.4 def & $16.4 \mathrm{a}$ & 0.0 & $0.73 d$ \\
\hline T. spelta (spelt wheat) & $A B D$ & 172 & 100.0 & $2.9 \mathrm{de}$ & $2.3 \mathrm{ef}$ & $5.8 \mathrm{e}$ & 0.0 & 0.13 ef \\
\hline
\end{tabular}

${ }^{1}$ Different letters, in each column, indicate statistical differences by the Duncan's Multiple Range Test $(p<0.05) ;{ }^{2}$ Percentage of initial explants produced callus; ${ }^{3}$ Percentage of initial explants produced embryogenic callus; ${ }^{4}$ Percentage of initial explants produced plantlets after the transferring to regeneration medium; ${ }^{5}$ Number of regenerated plantlets per one embryogenic callus; ${ }^{6}$ Average number of green plants regenerated per one initial explant.

mature embryos had a smaller size (Figure 2) and delayed differentiation.

The ability to form embryogenic calli depended on the wheat species and varied significantly even between wheats of the same ploidy (Tables 1 and 2). In this study, both immature and mature embryo-derived tissue cultures of tetraploid wheats with $A B$ genome generally had a greater embryogenic potential and efficiency of plant regeneration than the wheats with other genomic formulas. Efficiencies of immature embryo-derived cultures for AB-genome wheats ranged from $82.8 \%$ (T. turgidum) to $98.2 \%$ (T. polonicum). By the end of culture period, the entire explant surfaces of T. carthlicum, T. turgidum and T. polonicum were usually covered with numerous embryogenic structures (Figure 1C, 1D, 1E). Triticum dicoccum 'Runo' was distinguished by somatic embryos formation predominantly at the scutellum edge (Figure $1 \mathrm{H}$ ). The immature embryos of $A A B B D D$ hexaploid wheats formed a higher portion of nonembryogenic calli (Figure 1F, 1G, 1I, 1K), thus embryogenic callus induction ranged from only $20.2 \%$ (T. sphaerococcum) to $73.2 \%$ (T. spelta). Low frequencies were observed for wheats with $\mathrm{G}$ genome; where the percentage of embryogenic callus formation in tetraploid T.timopheevii was lower than in hexaploid T. kiharae (14.3\% and $36.7 \%$, respectively).

The diploid species $T$. monococcum and $T$. sinskajae were characterized by a similar pattern of embryogenic structure formation in the apical part of scutellum (Figure $1 \mathrm{~A}, \mathrm{~B})$, but they differed extremely in the ability to form embryogenic callus in immature-embryo-derived culture.
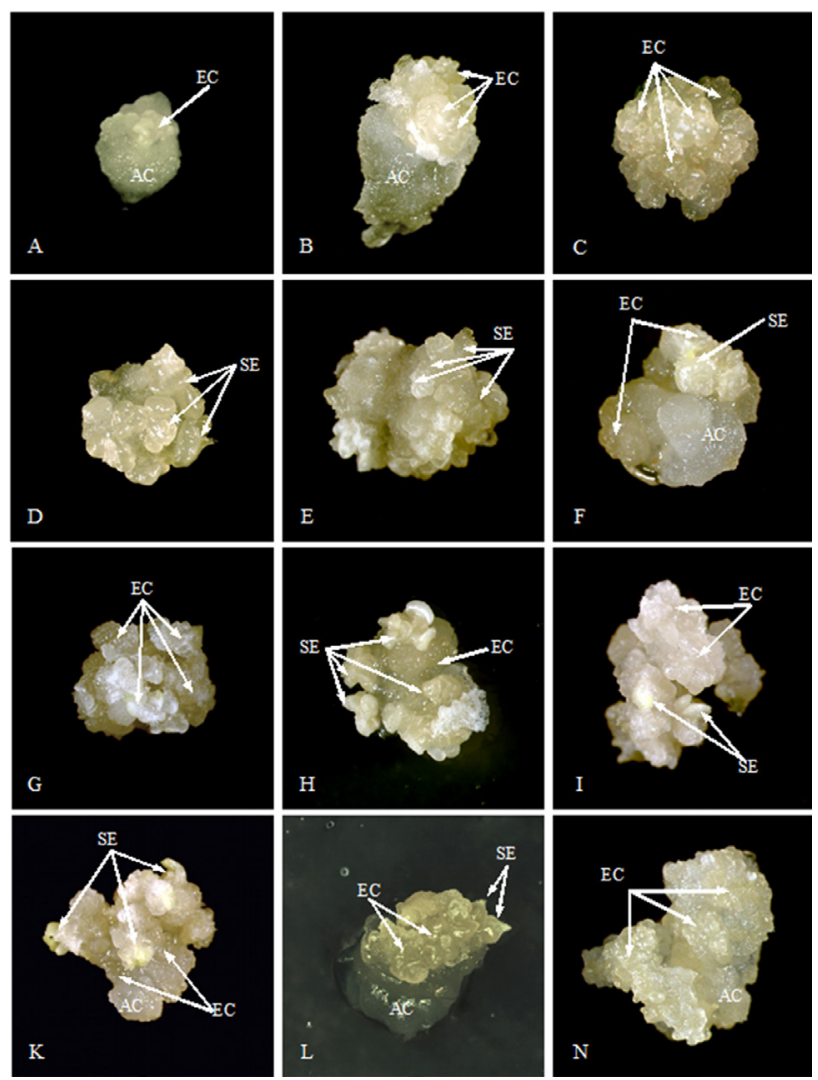

Figure 1. Embryogenic callus formation from immature embryo tissues after 4 weeks of culture in (A) T. monococcum, (B) T. sinskajae, (C) T. polonicum, (D) T. turgidum, (E) T. carthlicum, (F) T. compactum var. white, (G) T. compactum var. red, $(\mathrm{H})$ T. dicoccum 'Runo', (I) T. spelta, (K) T. sphaerococcum, (L) T. timopheevii, (N) T. kiharae, SE - somatic embryos, EC - embryogenic callus, $A C$ - amorphous (non-regenerating) callus. 
Triticum sinskajae, regarded as a natural naked mutation of T. monococcum (Goncharov 2011), has demonstrated a high ability to form embryogenic callus in immatureembryo-derived culture (83.5\%). In contrast, its progenitor T. monococcum showed the weakest in vitro response both in immature and mature embryo-derived tissue cultures. Explants of $T$. monococcum formed callus readily (92.2$97.8 \%)$, but their embryogenic capacity (0.7-2.8\%) and plant regeneration efficiency $(0.7-1.7 \%)$ were extremely low. This observation is in agreement with previous reports that showed either nonembryogenic callus formation or only occasional plant regeneration from cultured explants of T. monococcum (Lazar et al. 1983, Zale et al. 2004, Yin et al. 2011).

In this study, the in vitro performance of immature embryos of $T$. carthlicum (AABB genome) was best (14 green shoots per initial explant). Likewise, the culture efficiency of immature embryo tissues of the other wheat species with AABB genome ( $T$. dicoccum 'Runo', T. turgidum and T. polonicum) as well as of hexaploid $T$. spelta (AABBDD genome) was good (about 10-12 green plant per initial explants). This performance is usually regarded as sufficient for inclusion in genetic engineering programs for polyploid wheat cultivars (Pellegrineschi et al. 2002, He et al. 2010). The results of our study contradict findings of Yang at al.

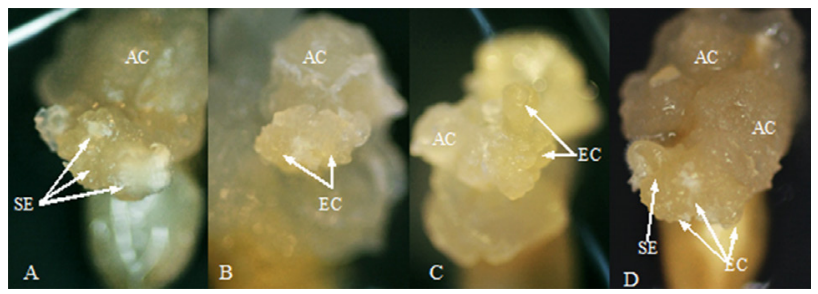

Figure 2. Embryogenic callus formation from mature embryo tissues after 4 weeks of culture in (A) T. kiharae, (B) T. carthlicum, (C) T. polonicum and (D) T. dicoccum, SE - somatic embryos, EC - embryogenic callus; AC - amorphous (non-regenerating) callus.

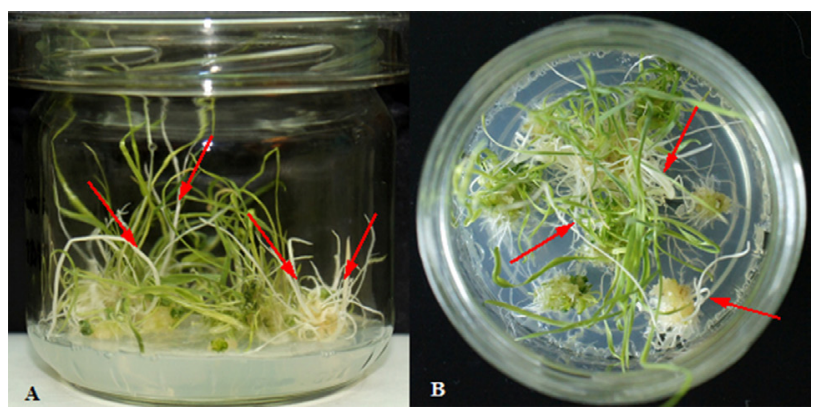

Figure 3. Regeneration of albino plants (indicated by arrows) from embryogenic callus of immature embryo tissues: (A) T. kiharae, (B) T. timopheevii. (2015), who reported that the immature embryo culture of

T. carthlicum, T. turgidum and T. polonicum displayed very low regeneration rates, while T. monococcum produced a high portion of differentiated callus. However, the results of this report are not directly comparable since other plant growth regulators/medium compositions were used and there are no data concerning the number of regenerated plants. In our study, the lowest culture efficiency (less than one green plant per initial explant) was observed for T. monococcum and T. timopheevii. This result was caused mainly by the insufficient capacity for embryogenesis and also by the albinism of regenerated plants.

Our studies revealed that six wheat species had produced albino-regenerants from immature tissue cultures, though in mature-tissue cultures this phenomenon was not observed. The higher portion of albino-regenerants was primarily attributed to G genomic formula wheats (Figure 3), such as T. kiharae (on average 8.5 albino regenerants per 100 plants) and T. timopheevii (on average 5.2 albino-regenerants per 100 plants). The other species, including T. carthlicum, T. compactum, T. monococcum, and T. sinskajae, produced a much lower number of albino plants (0.010.33 per embryogenic explant). In cereals, the regeneration of albino plants is a major challenge in routine anther and microspore culture experiments. For immature and mature embryo-derived cultures however, albinism is not a common problem. In androgenic cultures of wheat and barley, more than $60-80 \%$ of the variations associated with albino plant regeneration is genotype-dependent (Kumari et al. 2009, Makowska and Oleszczuk 2014). Nevertheless, the recovery of green vs. albino plants in anther cultures of cereals could be improved by optimized regeneration protocols (Kumari et al. 2009, Makowska and Oleszczuk 2014). In accordance with this assumption, our latest research showed that the changes in culture medium composition, namely of the concentrations/types of plant growth regulators and carbohydrate content, provided a more effective control of somatic embryogenesis and a lower production of albino plants in T. kiharae (Miroshnichenko et al. 2016).

Evidently, the two-step protocol used in our study to induce immature embryo-derived embryogenic callus of polyploid wheats with different genomes (T. monococcum, T. compactum, T. sphaerococcum, T. timopheevii, and T. kiharae) is not optimal. Moreover, some wheat germplasms with high efficiency of embryogenic callus formation (T. sinskajae, T. dicoccum, 
T. polonicum) formed less plantlets than the germplasms with low embryogenic ability (T. kiharae, T. compactum). These indicate that adequate changes in medium composition are required to achieve a satisfactory response at the different stages of somatic embryogenesis, including induction, maintenance and shoot development. For example, the application of the alternative 5-step protocol proposed by Eudes et al. (2003) involved a much larger number of media components/ growth regulators and resulted in satisfactory plant regeneration induction from scutellum of $T$. monococcum, $T$. durum and T. aestivum, without callus phase. There are numerous reports that describe the substantial increase in regeneration response in cultures of hexaploid bread wheat with AABBDD genome by modifications of the medium composition (Fennel et al. 1996, Machii et al. 1998, Barro et al. 1999, Bohorova et al. 2001, He and Lazzeri, 2001, Chauhan et al. 2007, Vendruscolo et al. 2008, Miroshnichenko et al. 2013). However, in most of the cited publications, the different manipulations were frequently inefficient in overcoming the strong genotypic effect and many varieties had to be screened to detect responsive wheat genotypes. Obviously, this indicates that no universal recipe can be given for an efficient culture of wheat species with different genomic formulas, and some optimization is likely required.

In contrast to the in vitro mature-embryo culture of other cereals, as of barley (He and Jia 2008) and rice (Li et al. 2015), the rate of somatic embryogenesis of the analyzed wheat species, with the exception of $T$. diccocum, was low, and they were unable to form more than one green plant per initial explant (Table 2). The callus regeneration ability of all cultures of $A B$ genome tetraploid wheats, except for T. turgidum, was higher (11.0-28.6\%) than that of hexaploid $(2.3-8.8 \%)$ and diploid wheats (0.7-6.1\%). Due to the relatively high frequency of embryogenic structure formation, T. diccocum 'Runo' had the highest culture efficiency of mature embryo tissues (3.41 plants/per initial explant). Generally speaking, this observation agrees with previous reports of $\mathrm{Bi}$ et al. (2007), who found that the response of mature embryo-derived callus formation of $T$. diccocum was better than that of the other tetraploid (T. durum) and hexaploid (T. aestivum) wheats. On the other hand, in several other reports (Chauhan et al. 2007, Chang et al. 2012, Yang et al. 2015 ) the difference between wheats with different genome composition (AABB vs. AABBDD) including T. diccocum was not clearly evident. In addition, conflicting data have been presented for diploid species. In this study as well as in that of Yin et al. (2011), the embryogenesis/regeneration ability of diploid wheats with AA genome was found to be very low. Recently, Özgen et al. (2015) reported that mature-embryo cultures of T. monococcum had a rather high callus regeneration capacity. However, in that study nodular calli with only green spots were taken into consideration, instead of the number of regenerated plants, so a correct comparison was not possible.

Mature wheat embryos are thought to be more recalcitrant to tissue culture than immature embryos, due to differences in the physiological and biochemical tissue status (Özgen et al. 1998, Filippov et al. 2006, Delporte et al. 2014, Yang et al. 2015). The acquisition of embryogenic competence in tissues of zygotic wheat embryos was shown to depend significantly on the endogenous level of growth regulators and total contents of phenols and soluble sugars (Jiménez and Bangerth 2001, Yang et al. 2015). With regard to the dormancy of the mature embryos at the stage of culture initiation, the particular interaction of the medium and other environmental parameters with genotype-specific endogenous factors was frequently required to achieve efficient morphogenesis, especially in endosperm-supported culture (Filippov et al. 2006, Bi et al. 2007, Chauhan et al. 2007, Yu et al. 2008, Miroshnichenko et al. 2011, Yin et al. 2011, Chang et al. 2012, Delporte et al. 2014). With the media tested here however, immature embryos are the preferable explant material. Regardless of the low culture efficiency of the studied germplasms, the ability of mature embryoderived cultures to generate entire green plants ought to be more deeply investigated, along with immature embryo and anther cultures. Currently, albinism still significantly delays breeding programs using doubled haploid technologies, so some comparative investigations may help discover genetic and developmental mechanisms responsible for the reduction of albino plant formation.

Thus, we conclude that conventional 2-step protocols using 2,4-D as main exogenous inducer can be used to generate a readily sustainable supply of morphogenic material from immature embryos of T. carthlicum, T. dicoccum, T. polonicum, T. spelta, and T. turgidum. On the other hand, the endosperm-supported protocol, which is originally developed for mature embryos of bread wheat, might fail to meet the specific requirements of wheat species with various genetic backgrounds to provide sufficient plant regeneration. Some modifications of protocols should however be performed, e.g., of the type and concentration of micronutrients, growth regulators, sugars, and organic compounds in the medium. This is particularly true for cultures of diploid (T. monococcum, T. sinskajae), hexaploid (T. compactum, T. sphaerococcum) and polyploid germplasms with G chromosomes (T. timopheevii, T. kiharae), to provide a more effective control of somatic 
Tissue culture efficiency of wheat species with different genomic formulas

embryogenesis and to improve the quality of regenerated plants for developing efficient recombinant DNA techniques.

\section{ACKNOWLEDGEMENTS}

The authors thank Prof. Ludmila A. Bespalova, Krasnodar Lukyanenko Research Institute of Agriculture and Prof. Gennady I. Karlov, Center for Molecular Biotechnology, Russian State Agrarian University - Moscow Timiryazev Agricultural Academy, for kindly providing the seeds of various wheat germplasms.

\section{REFERENCES}

Barro F, Martin A, Lazzeri PA and Barceló P (1999) Medium optimization for efficient embryogenesis and plant regeneration from immature inflorescences and immature scutella of elite cultivars of wheat, barley and tritordeum. Euphytica 108: 161-167.

Bi RM, Kou M, Chen LG, Mao SR and Wang HG (2007) Plant regeneration through callus initiation from mature embryo of Triticum. Plant Breeding 126: 9-12.

Bohorova NE, Pfeiffer WH, Mergoum M, Crossa J, Pacheco M and Estanol $P$ (2001) Regeneration potential of CIMMYT durum wheat and triticale varieties from immature embryos. Plant Breeding 120: 291-295.

Chang CM, Penna S and Bhagwat SG (2012) Callus induction and plant regeneration from different Triticum species. The Asian and Australasian Journal of Plant Science and Biotechnology 6: 56-62.

Chauhan H, Desai SA and Khurana P (2007) Comparative analysis of the differential regeneration response of various genotypes of Triticum aestivum, Triticum durum and Triticum dicoccum. Plant Cell Tissue and Organ Culture 91: 191-199.

Delporte F, Pretova A, du Jardin P and Watillon B (2014) Morpho-histology and genotype dependence of in vitro morphogenesis in mature embryo cultures of wheat. Protoplasma 251: 1455-1470.

Eapen S and Rao PS (1982) Plant regeneration from callus cultures of durum and emmer wheat. Plant Cell Reports 1: 215-218.

Eudes F, Acharya S, Laroche A, Selinger LB and Cheng KJ (2003) A novel method to induce direct somatic embryogenesis, secondary embryogenesis and regeneration of fertile green cereal plants. Plant Cell Tissue and Organ Culture 73: 147-157.

Fennell S, Bohorova N, Ginkel MV, Crossa J and Hoisington D (1996) Plant regeneration from immature embryos of 48 elite CIMMYT bread wheats. Theoretical and Applied Genetics 92: 163-169.

Filippov M, Miroshnichenko D, Vernikovskaya D and Dolgov S (2006) The effect of auxins, time exposure to auxin and genotypes on somatic embryogenesis from mature embryos of wheat. Plant Cell Tissue and Organ Culture 84: 213-222.

Goncharov NP (2011) Genus Triticum L. taxonomy: the present and the future. Plant Systematics and Evolution 295: 1-11.

He GY and Lazzeri PA (2001) Improvement of somatic embryogenesis and plant regeneration from durum wheat (Triticum turgidum var. durum Desf.) scutellum and inflorescence cultures. Euphytica 119: 369-376.

He TF and Jia JF (2008) High frequency plant regeneration from mature embryo explants of highland barley (Hordeum vulgare L. var. nudum Hk. f.) under endosperm-supported culture. Plant Cell Tissue and Organ Culture 95: 251-254.

He Y, Jones HD, Chen S, Chen XM, Wang DW, Li KX, Wang DS and Xia LQ (2010) Agrobacterium-mediated transformation of durum wheat (Triticum turgidum L. var. durum cv Stewart) with improved efficiency. Journal of Experimental Botany 61: 1567-1581.

Jiménez VM and Bangerth F (2001) Endogenous hormone concentrations and embryogenic callus development in wheat. Plant Cell Tissue and Organ Culture 67: 37-46.

Jones HD (2015) Wheat biotechnology: current status and future prospects. In Azhakanandam K, Silverstone A, Daniell H and Davey RM (eds) Recent advancements in gene expression and enabling technologies in crop plants. Springer New York, New York, p. 263-290.

Kumari M, Clarke, Small I and Siddique KHM (2009) Albinism in plants: a major bottleneck. Critical Reviews in Plant Science 28: 393-409.

Lazar MD, Collins GB and Vian WE (1983) Genetic and environmental effects on the growth and differentiation of wheat somatic cell cultures. Journal of Heredity 74: 353-357.

Li D, Xu H, Sun X, Cui Z, Zhang Y, Bai Y, Wang X and Chen W (2015) Differential transformation efficiency of Japonica rice varieties developed in northern China. Crop Breeding and Applied Biotechnology 15: 162-168.

Longin CF and Reif JC (2014) Redesigning the exploitation of wheat genetic resources. Trends in Plant Science 19: 631-636.

Machii H, Mizuno H, Hirabayashi T and Hagio T (1998) Screening wheat genotypes for high callus induction and regeneration capability from anther and immature embryo cultures. Plant Cell Tissue and Organ Culture 53: 67-74.

Makowska K, Oleszczuk S (2014) Albinism in barley androgenesis. Plant Cell Reports 33: 385-392.

Mamrutha HM, Kumar R, Venkatesh K, Sharma P, Kumar R, Tiwari V and Sharma I (2014) Genetic transformation of wheat - Present status and future potential. Journal of Wheat Research 6: 107-119.

Miroshnichenko DN, Filippov MV and Dolgov SV (2013) Medium optimization for efficient somatic embryogenesis and in vitro plant regeneration of spring common wheat varieties. Russian Agricultural Sciences 39: 24-28.

Miroshnichenko DN, Poroshin G N and Dolgov SV (2011) Genetic transformation of wheat using mature seed tissues. Applied 
Biochemistry and Microbiology 47: 767-775.

Miroshnichenko D, Chernobrovkina M and Dolgov S (2016) Somatic embryogenesis and plant regeneration from immature embryos of Triticum timopheevii Zhuk. and Triticum kiharae Dorof. et Migusch, wheat species with $\mathrm{G}$ genome. Plant Cell Tissue and Organ Culture 125: 495-508.

Murashige T and Skoog $F$ (1962) A revised medium for rapid growth and bioassays with tobacco tissue cultures. Physiology Plantarum 15: $473-497$.

Nevo E (2011) Triticum. In Kole C (ed) Wild crop relatives: genomic and breeding resources, cereals. Springer Verlag, Berlin, p. 407-456.

Özgen M, Türet M, Altmok S and Sancak C (1998) Efficient callus culture induction and plant regeneration from mature embryo culture of winter wheat (Triticum aestivum L) genotypes. Plant Cell Reports 18: 331-335.

Özgen M, Birsin MA and Benlioglu B (2015) Biotechnological characterization of a diverse set of wheat progenitors (Aegilops sp. and Triticum sp.) using callus culture parameters. Plant Genetic Resources, First View: 1-6, Published online: 14 August 2015, DOI: 10.1017/S1479262115000350.

Pellegrineschi A, Noguera LM, Skovmand B, Brito RM, Velazquez L, Salgado MM, Hernandez R, Warburton M and Hoisington D (2002)
Identification of highly transformable wheat genotypes for mass production of fertile transgenic plants. Genome 45: 421-430.

Repellin A, Baga M, Jauhar PP and Chibbar RN (2001) Genetic enrichment of cereal crops via alien gene transfer: new challenges. Plant Cell Tissue and Organ Culture 64: 159-183.

Vendruscolo ECG, Schuster I, Negra ES and Scapim CA (2008) Callus induction and plant regeneration by Brazilian new elite wheat genotypes. Crop Breeding and Applied Biotechnology 8: 195-201.

Yang S, Xu K, Wang Y, Bu B, Huang W, Sun F, Liu S and Xi Y (2015) Analysis of biochemical and physiological changes in wheat tissue culture using different germplasms and explant types. Acta Physiology Plantarum 37: 1-10.

Yin GX, Wang YL, She MY, Du LP, Xu HJ, MA JX and Ye XG (2011) Establishment of a highly efficient regeneration system for the mature embryo culture of wheat. Agricultural Sciences in China 10: 9-17.

Yu Y, Wang J, Zhu ML and Wei ZM (2008) Optimization of mature embryobased high frequency callus induction and plant regeneration from elite wheat cultivars grown in China. Plant Breeding 127: 249-255.

Zale JM, Borchardt-Wier H, Kidwell KK and Steber CM (2004) Callus induction and plant regeneration from mature embryos of a diverse set of wheat genotypes. Plant Cell Tissue and Organ Culture 76: 277-281. 